\title{
Risk assessment models for potential use in the emergency department have lower predictive ability in older patients compared to the middle-aged for short-term mortality - a retrospective cohort study
}

Martin Schultz ${ }^{1,2^{*}}$ (D), Line Jee Hartmann Rasmussen ${ }^{3}$, Nicolas Carlson ${ }^{4}$, Rasmus Bo Hasselbalch ${ }^{1}$, Birgitte Nybo Jensen ${ }^{5}$, Lotte Usinger ${ }^{2}$, Jesper Eugen-Olsen ${ }^{3}$, Christian Torp-Pedersen ${ }^{6,7}$,

Lars Simon Rasmussen ${ }^{8}$ and Kasper Karmark Iversen ${ }^{1}$

\begin{abstract}
Background: Older patients is a complex group at increased risk of adverse outcomes compared to younger patients, which should be considered in the risk assessment performed in emergency departments. We evaluated whether the predictive ability of different risk assessment models for acutely admitted patients is affected by age.

Methods: Cohort study of middle-aged and older patients. We investigated the accuracy in discriminating between survivors and non-survivors within 7 days of different risk assessment models; a traditional triage algorithm, a triage algorithm with clinical assessment, vital signs, routine biomarkers, and the prognostic biomarker soluble urokinase plasminogen activator receptor (suPAR).

Results: The cohort included 22,653 (53.2\%) middle-aged patients (age 40-69years), and 19,889 (46.8\%) older patients (aged 70+ years). Death within 7 days occurred in 139 patients (0.6\%) in middle-aged patients and 596 (3.0\%) of the older patients. The models based on vital signs and routine biomarkers had the highest area under the curve (AUC), and both were significantly better at discriminating 7-day mortality in middle-aged patients compared to older patients; AUC (95\% Cl): 0.88 (0.84-0.91), 0.75 (0.72-0.78), $P<0.01$, and $0.86(0.82-0.90), 0.76(0.73-0.78), P<0.001$. In a subgroup of the total cohort (6.400 patients, $15.0 \%)$, the suPAR level was available. sUPAR had the highest AUC of all individual predictors with no significant difference between the age groups, but further research in this biomarker is required before it can be used.
\end{abstract}

(Continued on next page)

\footnotetext{
* Correspondence: Martin.schultz@regionh.dk

'Department of Cardiology, Herlev and Gentofte Hospital, University of

Copenhagen, Herlev Ringvej 75, DK-2730 Herlev, Denmark

2Department of Internal Medicine and Geriatrics, Herlev and Gentofte

Hospital, University of Copenhagen, Herlev, Denmark

Full list of author information is available at the end of the article
}

(c) The Author(s). 2019 Open Access This article is distributed under the terms of the Creative Commons Attribution 4.0 International License (http://creativecommons.org/licenses/by/4.0/), which permits unrestricted use, distribution, and reproduction in any medium, provided you give appropriate credit to the original author(s) and the source, provide a link to the Creative Commons license, and indicate if changes were made. The Creative Commons Public Domain Dedication waiver (http://creativecommons.org/publicdomain/zero/1.0/) applies to the data made available in this article, unless otherwise stated. 


\begin{abstract}
(Continued from previous page)
Conclusion: The predictive value was lower in older patients compared to middle-aged patients for all investigated models. Vital signs or routine biomarkers constituted the best models for predicting 7-day mortality and were better than the traditional triage model. Hence, the current risk assessment for short-term mortality can be strengthened, but modifications for age should be considered when constructing new risk assessment models in the emergency department.
\end{abstract}

Keywords: Emergency department, Older patients, Risk assessment, Triage

\section{Background}

Emergency departments (EDs) must prioritise treatment of patients of all ages and health status according to urgency while counterbalancing the negative effects of ED crowding on patient safety $[1,2]$. EDs commonly employ triage algorithms to assess risk and prioritise according to the perceived urgency of patients' conditions. These algorithms typically consist of a combination of vital signs and primary complaints for risk assessment [3].

Older patients aged 65 years and older account for 12 $24 \%$ of all ED visits; additionally, both the number of visits and the utilisation of resources in the ED are increasing $[4,5]$. Compared to younger patients, the older often have a greater burden of comorbidities, more severe illnesses $[4,6,7]$, and often present with atypical symptoms and nonspecific complaints $[4,8,9]$. Therefore, older patients constitute a complex group at high risk; within 3 months following an ED visit 20\% of older patients had been hospitalised, 20\% revisited the ED, and approximately 5\% were dead [5]. Additionally, age-related changes in physiology may result in reduced variability and decreased response to stress [10], rendering vital signs less useful for assessment of urgency and severity of acute illness in the older [10, 11]. However, none of the currently used triage algorithms stratifies patients according to age [12].

Risk assessment in the ED can be done using one of the traditional triage algorithms [12], but recent studies have attempted to improve risk assessment using different approaches or adding new elements. Incorporation clinical assessment is one possibility as physicians first clinical impression is associated with morbidity and mortality, and because ED staff using only clinical intuition can identify patients at risk of death [13, 14]. Different vital signs are associated with adverse outcome and using these in risk scores or simply the number of abnormal vital signs can identify patients at risk, providing a second approach for a better risk assessment $[15,16]$. A third possibility, is risk assessment using the routine biomarkers analysed from blood samples during acute admission. Many of these biomarkers have value in risk stratification, in addition to their diagnostic value. For instance $\mathrm{C}$-reactive protein (CRP) and albumin are associated with mortality, $[17,18]$ and a statistical model containing eight routine biomarkers has been shown to be significantly better in predicting mortality compared to traditional triage [19]. Finally, newly discovered experimental biomarkers have been found to carry prognostic information that allows for an accurate risk discrimination [20-22]. One of these prognostic biomarkers is soluble urokinase plasminogen activator receptor (suPAR), which is associated with disease severity of several acute and chronic conditions, and with length of stay, readmissions and mortality in ED patients [23-25].

The optimal and best approach, however, remains undetermined. The aim of this study was to evaluate the predictive ability of different models for risk assessment regarding short-term mortality in ED patients and to compare the accuracy of the models in middle-aged and older patients.

\section{Methods}

\section{Study design}

For this study of middle-aged and older patients presenting at an ED, we used data from the TRIAGE II and TRIAGE III studies [26, 27]. In brief, the TRIAGE II study was a prospective, interventional cluster-randomised trial comparing a newly developed triage algorithm, which adds a clinical assessment to a traditional triage algorithm [27]. The TRIAGE III study was a cluster-randomised interventional trial investigating the effect of introducing the nonspecific prognostic biomarker soluble urokinase plasminogen activator receptor (suPAR) as a routine blood test in the ED [26, 28].

\section{Setting}

TRIAGE II and TRIAGE III included ED patients at Bispebjerg Hospital and Herlev Hospital, Denmark; both are University hospitals with 24-h acute care of patients, offering all internal medicine specialities, general and orthopaedic surgery, intensive care, and level- 2 trauma care.

\section{Patients}

Patients' first ED visit from the TRIAGE studies was included, subsequent readmissions were excluded. Scientific research use different age cut-offs to define older patients ranging from 60 to 75 years [4], in the present study we classified patients aged 40 to 69 years as middle-aged, and patients aged 70+ years as older, in accordance with 
previous research from emergency departments [29-31]. Patients younger than 40 years were excluded. The TRIAGE studies included ED patients from the same locations in a continuous time period making the study populations similar. To assess the different risk assessment models, we combined the two study populations into one large cohort for this study.

\section{Data collection}

Vital signs, triage categories, and results of blood tests were recorded at the ED visit. Vital signs, blood tests and triage categories were retrieved from local hospital databases. Follow-up data on hospital admissions, discharges, and diagnoses were obtained from the Danish National Patient Registry, [32, 33] and vital status at the end of each TRIAGE study was obtained from the Danish Civil Registration System, using patients' unique Danish civil registration number.

\section{Outcome measures}

Outcomes were all-cause mortality within two and 7 days.

\section{Risk assessment models}

We assessed five different types of risk assessment models in the ED; I) a traditional triage algorithm, II) a triage algorithm with clinical assessment, III) vital signs, IV) routine biomarkers, and finally, V) the experimental biomarker suPAR, and compared the predictive abilities of middle-aged patients to older patients.

\section{Traditional triage algorithm}

The commonly used triage algorithm in Denmark is a local adaptation of "Adaptive Process Triage" (ADAPT), [34] which is based on vital signs and the presenting complaint $[35,36]$. Patients are divided into five categories: Red (most urgent), Orange, Yellow, Green (least urgent), and Blue (minor injuries). In this analysis, patients triaged "Green" and "Blue" were combined. ADAPT is similar to other traditional 5-level triage algorithms [12].

\section{Triage with clinical assessment}

"The Copenhagen Triage algorithm" (CTA) is a newly developed triage algorithm with five categories (similar to ADAPT) based on measurement of vital signs and supplemental of oxygen, followed by a systematic clinical assessment by the ED staff, permitting modification of the final triage category [27].

\section{Vital signs}

The vital signs; heart rate, respiratory rate, arterial oxygen saturation, systolic blood pressure, and temperature were measured at arrival to the ED.

\section{Routine biomarkers}

In the present study, we included levels of albumin, CRP, creatinine, haemoglobin, leukocyte count, sodium, potassium, and platelets, as they are routinely measured during acute admissions and were available.

\section{Experimental biomarker}

In recent years, several new biomarkers with prognostic information have been discovered as promising risk assessment tools for use in the ED [37]. suPAR is a nonspecific biomarker of inflammation associated with mortality [21, 24].

\section{Statistics}

Continuous variables are presented with median and interquartile range (IQR) or mean (standard deviation), and categorical variables as number ( $\mathrm{n}$ ) and percentages (\%). Baseline characteristics were compared using the chi-square test, Student's two-sample t-test, and Wilcoxon rank-sum test. The ability of triage, vital signs, and biomarkers to discriminate on mortality was assessed using the area under the curve (AUC) for receiver operating characteristics curves. AUCs were compared using the DeLong's method [38]. Triage categories, vital signs, and biomarkers were used as continuous variables in logistic regression to calculate AUCs. Combined models were created using multiple logistic regression. Patients with missing predictors were not included in the respective analyses. A $P$-value below 0.05 was considered statistically significant. Statistical analyses were performed in $\mathrm{R}$ version 3.3.3 [39] using the pROC package [40].

\section{Results}

\section{Study population}

The TRIAGE II study included ED visits of 39,883 patients from March 1, 2015, to January 31, 2016, and the TRIAGE III study included ED visits of 16,801 patients from January 11, 2016, to June 6, 2016. After exclusion of patients younger than 40 years, the combined cohort consisted of 42,542 patients (75.1\%). The mean (SD) age was $67.2(14.9)$ years, and $21,880(51.4 \%)$ were women. At inclusion, 22,653 (53.2\%) were age $40-69$ years (middle-aged), and 19,889 (46.8\%) were aged $70+$ years (older). Death from all causes within 7 days occurred in 139 patients $(0.6 \%)$ in the group of middle-aged patients and in $596(3.0 \%)$ in the group of older patients. All baseline characteristics were significantly different when comparing the two age-groups, except for temperature, Table 1. The two TRIAGE populations were comparable regarding age, sex, and outcomes, but minor, but statistically significant, differences in the levels of the biomarkers were observed (Additional file 1: Table S1). 
Table 1 Baseline characteristics at first visit at the emergency department. Patients are grouped according to their age; 40-69 years (middle-aged) and $70+$ years (older)

\begin{tabular}{|c|c|c|c|}
\hline & $\begin{array}{l}\text { Middle-aged } \\
N=22,653\end{array}$ & $\begin{array}{l}\text { Older } \\
N=19,889\end{array}$ & $P$ \\
\hline Female sex, n (\%) & $10,687(47.2)$ & $11,193(56.3)$ & $<0.001$ \\
\hline Age (years), mean (SD) & $55.5(8.6)$ & $80.7(7.4)$ & \\
\hline Triage: ADAPT & $11,389(50.3)$ & $9746(49.0)$ & \\
\hline Triage category: Red, n (\%) & $418(3.1)$ & $482(4.9)$ & $<0.001$ \\
\hline Triage category: Orange, n (\%) & $3376(24.0)$ & $3038(31.2)$ & 0.02 \\
\hline Triage category: Yellow, n (\%) & $3953(36.3)$ & $3428(35.1)$ & 0.49 \\
\hline Triage category: Green, n (\%) & $3642(36.5)$ & $2798(28.7)$ & $<0.001$ \\
\hline Triage: CTA & $7983(35.2)$ & $6655(33.5)$ & \\
\hline Triage category: Red, n (\%) & $189(2.4)$ & $225(3.4)$ & $<0.001$ \\
\hline Triage category: Orange, n (\%) & $1276(16.0)$ & $1143(17.2)$ & 0.05 \\
\hline Triage category: Yellow, n (\%) & $3092(38.7)$ & $2409(36.2)$ & 0.01 \\
\hline Triage category: Green, n (\%) & $3426(42.9)$ & $2878(43.2)$ & 0.70 \\
\hline Vital signs & $13,200(58.3)$ & $10,992(55.3)$ & \\
\hline Heart rate (beats/min), mean (SD), & $86(20)$ & $85(20)$ & $<0.001$ \\
\hline Arterial oxygen saturation (\%), median (IQR) & 97 (96-99) & $96(95-98)$ & $<0.001$ \\
\hline Respiratory rate (breaths/min), mean (SD) & $17(3)$ & $18(4)$ & $<0.001$ \\
\hline Systolic blood pressure (mmHg), mean (SD) & $140(25)$ & $146(29)$ & $<0.001$ \\
\hline Temperature $\left({ }^{\circ} \mathrm{C}\right)$, mean $(\mathrm{SD})$ & $36.8(0.8)$ & $36.8(0.8)$ & 0.07 \\
\hline Biomarkers levels, n (\%) & $13,032(57.5)$ & $13,039(65.6)$ & \\
\hline Albumin (g/L), median (IQR) & $41(37-47)$ & $38(34-41)$ & $<0.001$ \\
\hline Creatinine $(\mu \mathrm{mol} / \mathrm{L})$, median (IQR) & $72(60-87)$ & $83(66-111)$ & $<0.001$ \\
\hline CRP (mg/L), median (IQR) & $4(3-33)$ & $11(3-61)$ & $<0.001$ \\
\hline Haemoglobin (mmol/L), median (IQR) & $8.6(7.8-9.2)$ & $7.9(7.0-8.6)$ & $<0.001$ \\
\hline Leucocytes ( $\left.\times 10^{9} / \mathrm{L}\right)$, median (IQR) & $8.6(6.6-11.3)$ & $8.9(6.8-11.8)$ & $<0.001$ \\
\hline Potassium (mmol/L), median (IQR), & $3.9(3.7-4.2)$ & $4.0(3.7-4.3)$ & $<0.001$ \\
\hline Sodium (mmol/L), median (IQR) & $139(136-141)$ & $138(135-141)$ & $<0.001$ \\
\hline Platelets $\left(\times 10^{9} / \mathrm{L}\right)$, median (IQR) & $245(200-300)$ & $242(195-309)$ & $<0.001$ \\
\hline suPAR (ng/mL), median (IQR) & $3.7(2.8-5.2)$ & $5.4(4.0-7.5)$ & $<0.001$ \\
\hline \multicolumn{4}{|l|}{ Mortality } \\
\hline Mortality within 2-days, n (\%) & $73(0.3)$ & $263(1.3)$ & $<0.001$ \\
\hline Mortality within 7-days, n (\%) & $139(0.6)$ & $596(3.0)$ & $<0.001$ \\
\hline
\end{tabular}

CRP C-reactive protein, IQR Interquartile range, SD Standard deviation, suPAR Soluble urokinase plasminogen activator receptor

\section{Predictive ability of different risk assessment models}

AUCs for 2-day and 7-day mortality for all five risk assessment models stratified in age-groups are presented in Table 2, individual vital signs and biomarkers in Additional file 1: Table S2 and the AUCs regarding 7-day mortality is presented in Additional file 1: Figure S1.

\section{Traditional triage algorithm}

ADAPT triage categories were recorded for 11,389 (50.3\%) of middle-aged patients and 9746 (49.0\%) of older patients. Significantly fewer middle-aged patients were triaged "Red" compared to the older, while more were triaged "Green" (Table 1). No difference in the predictive ability of short-term, all-cause mortality was observed between the two groups (Table 2):

\section{Triage with clinical assessment}

CTA triage categories were recorded for 7983 (35.2\%) of middle-aged patients and 6655 (33.5\%) of older patients. Comparison of the distribution in triage categories showed significantly more middle-aged patients in the "Yellow" category and less in the "Red" (Table 1). AUC analyses showed better prognostic ability compared to 
Table 2 Comparison of AUCs in predicting short-term mortality of patients acutely presenting at the emergency department, grouped according to age: 40-69 years (middle-aged), and 70+ years (older)

\begin{tabular}{llll}
\hline AUC, 95\% Cl & Middle-aged & Older & $P$ \\
& $N=22,653$ & $N=19,889$ & \\
\hline ADAPT, 2-day mortality & $0.80(0.73-0.87)$ & $0.76(0.72-0.81)$ & 0.40 \\
ADAPT, 7-day mortality & $0.72(0.66-0.78)$ & $0.71(0.67-0.74)$ & 0.69 \\
CTA, 2-day mortality & $0.83(0.77-0.89)$ & $0.78(0.73-0.84)$ & 0.22 \\
CTA, 7-day mortality & $0.79(0.73-0.85)$ & $0.73(0.69-0.76)$ & 0.09 \\
Vital signs, 2-day mortality & $0.89(0.84-0.94)$ & $0.81(0.77-0.86)$ & 0.02 \\
Vital signs, 7-day mortality & $0.88(0.84-0.91)$ & $0.75(0.72-0.78)$ & $<0.001$ \\
Biomarkers, 2-day mortality & $0.84(0.78-0.90)$ & $0.75(0.71-0.79)$ & 0.02 \\
Biomarkers, 7-day mortality & $0.86(0.82-0.89)$ & $0.76(0.73-0.78)$ & $<0.001$ \\
suPAR, 2-day mortality & $0.82(0.66-0.97)$ & $0.73(0.64-0.81)$ & 0.32 \\
suPAR, 7-day mortality & $0.82(0.73-0.91)$ & $0.77(0.72-0.82)$ & 0.32 \\
\hline
\end{tabular}

ADAPT Adaptive process triage, AUC Area under the curve, $C I$ Confidence interval, CRP C-reactive protein, CTA Copenhagen triage algorithm, suPAR Soluble urokinase plasminogen activator receptor, Vitals: Prediction model using four vital signs (hear rate, oxygen saturation, respiratory rate, systolic blood pressure), Biomarker: Predictive model using levels of seven routine biomarkers (albumin, creatinine, c-reactive protein, haemoglobin, leucocytes, potassium, sodium)

ADAPT, and no difference in the prognostic ability between age groups (Table 2).

\section{Vital signs}

A complete set of the five included vital signs was recorded in 13,200 (58.3\%) of middle-aged- and 10,992 $(55.3 \%)$ of older patients. Of five available vital signs, four had a predictive value of 2-day and 7-day mortality (not temperature). Of the remaining vital signs, the respiratory rate had the highest AUC and was significantly better at predicting short-term mortality among the middle-aged than among the older (Additional file 1: Table S2). Combining the vital signs (heart rate, arterial oxygen saturation, systolic blood pressure, and respiratory rate) in a prediction model showed significantly better predictive ability for short-term mortality among the middle-aged compared to the older (Table 2).

\section{Routine biomarkers}

Included routine biomarkers measured at ED visit were available in 13,032 (57.5\%) of the middle-aged and $13,039(65.6 \%)$ of the older. All routine biomarkers, except for platelets, had an individual predictive value of 2-day and 7-day mortality in both groups. Levels of albumin, CRP, haemoglobin, and sodium were significantly better at predicting short-term mortality in the middle-aged patients (Additional file 1: Table S2). A combined model consisting of seven biomarkers (platelets not included) had significantly higher AUC in predicting short-term mortality in the group of middle-aged patients compared to the older (Table 2).

\section{Experimental biomarker}

suPAR was available in 3227 (14.2\%) middle-aged- and $3173(16.0 \%)$ of the older patients. Its predictive ability was higher than individual vital signs or routine biomarkers and comparable to the triage algorithm (Additional file 1: Table S2). There was no difference observed among age-groups (Table 2).

\section{Overall predictive ability across age deciles}

All-cause mortality within 7 days was increasing with increasing age. Stratifying the study population according to age deciles decreased the accuracy in all five risk assessment models, Fig. 1. In the two triage algorithms, this was not significant. The AUCs were significantly lower in the models consisting of vital signs, biomarkers, and suPAR when comparing age 50 and age 80 years, $p<0.001$.

\section{Discussion}

In this study of 42,452 acutely presenting patients, we found that risk assessment models based on vital signs and routine biomarkers, had the best predictive abilities compared to the other risk assessment models tested; both models were significantly better at discriminating on short-term mortality in middle-aged patients compared to older patients. Furthermore, all five risk assessment models included in this study showed fair to good abilities in discriminating between survivors and non-survivors at 7 days, but lower AUCs for the older.

The traditional triage algorithm (ADAPT), which is commonly used, was less accurate than the other models but did not differ significantly between age groups. Most of the included vital signs and biomarkers had individual predictive value but combining them in models yielded the highest accuracy. Among the individual routine biomarkers, albumin and CRP had the highest AUCs and performed significantly better in the middle-aged patients than in older patients. The experimental biomarker, suPAR, had the highest AUC of all investigated individual predictors, and there was no significant difference in the predictive value between the age groups. However, suPAR was only available in $14.2 \%$ of the middle-aged patient and in $16.0 \%$ of the older patients and only in patients from the TRIAGE III study, representing a small subgroup of the total cohort, which warrants further internal and external validation of the predictive ability before it can be considered for use in the ED. Importantly, our results demonstrate the instrumental value of employing vital signs as part of risk assessment at triage, which previously have been demonstrated to predict mortality, $[15,41]$ but the 


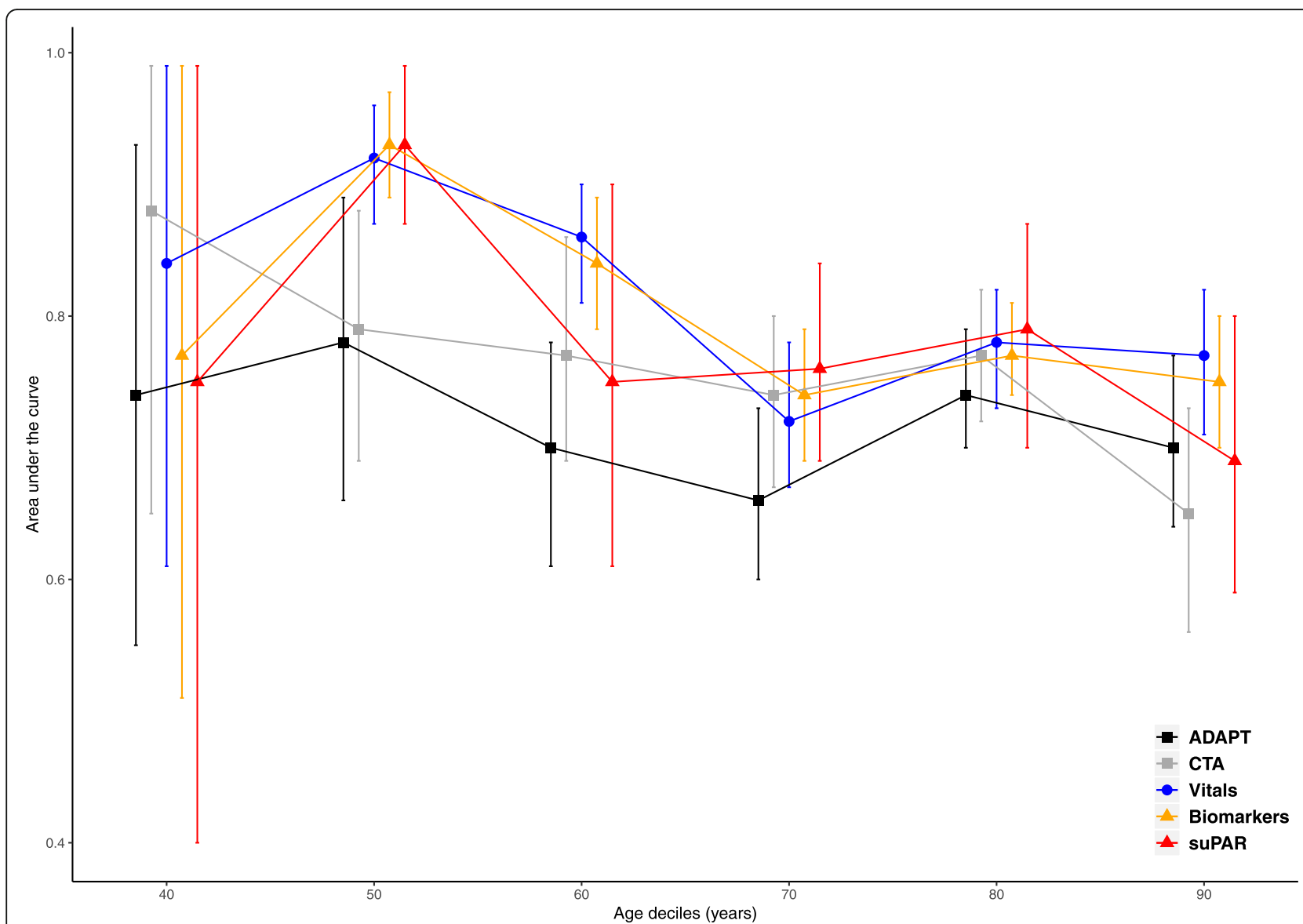

Fig. 1 Area under the Curve for Receiver operating characteristics for all-cause mortality within 7 days for acutely admitted patients. The graph presents five different approaches to risk assess patients acutely presenting at the emergency department. Patients are stratified in age deciles according to their age at the first visit. The five approaches include; Two different triage algorithms; Adaptive Process Triage (ADAPT) and Copenhagen Triage Algorithm (CTA), a predictive model using four vital signs (heart rate, arterial oxygen saturation, respiratory rate and systolic blood pressure), a predictive model using levels of seven routine biomarkers (albumin, creatinine, c-reactive protein, haemoglobin, leucocytes, potassium, sodium), and the experimental biomarker soluble urokinase plasminogen activator receptor (suPAR). Mortality in age deciles; $40: 0.2 \%$, 50: $0.4 \%, 60: 1.0 \%, 70: 1.9 \%, 80: 3.6 \%, 90: 5.2 \%$

predictive value is not equal across age groups. Vital signs have previously shown to be less predictive in the older prior to cardiac arrest and at triage [11, 42]. Additionally, vital signs have increased sensitivity when observed as serial measurements and might provide the best information with an individualised reference range, [10] however, this is not viable in the ED. Constructing age-dependent reference ranges or cut-offs for use in triage would be an alternative solution. We also demonstrate that use of routine biomarkers in risk assessment models could improve discriminative ability, but transference of these models directly into clinical use remains complicated. The performance of the investigated models in this study is comparable to other risk assessment tools of mortality assessed in a systematic review of scores based on different composition of vital signs and biomarkers [43]. In the cohort of middle-aged patients, our models of vital signs and biomarkers were comparable to the best performing tools in the review, which both used age and incorporated either vital signs or biomarkers.

Current triage algorithms have been validated to a limited extent in older patients, [3] and there is little or no evidence regarding performance in older patients or using age-adjustment among the most commonly used triage algorithms [12]. Only the Emergency Severity Index (ESI) algorithm has been investigated with regard to older patients, results were however conflicting [44-46]. Interestingly, the inclusion of age has been proposed in different trackand-trigger systems and predictions models, based on a perceived augmentation of predictive accuracy [47-51]. Furthermore, the physiological scoring systems, Simplified Acute Physiology Score (SAPS II) [52] and Acute Physiology And Chronic Health Evaluation (APACHE) [51], which are used for risk stratification of severity and risk of mortality in intensive care, include age. Finally, important 
factors affecting outcome for older patients are functional and cognitive status and comorbidity [53]. Incorporating these factors may increase the accuracy of the triage algorithm and lead to better management and treatment of older patients.

In summary, risk assessment can be strengthened using one of the described models, however, modifications for patients over 70 years of age should be considered. Our results indicate that incorporating age may improve the accuracy of risk assessment in older patients in future triage, but factors like functional and cognitive status should also be considered. Furthermore, new models should be validated in external cohorts before implementation. The experimental biomarker suPAR shows promise in the current study, but the results can only be considered as hypothesis-generation. Further research in this biomarker, the potential use, and possible interventions to elevated levels is warranted. Whether a new or redesigned triage algorithm will lead to better management and prognosis of older patients will require further prospective interventional studies.

\section{Strengths and limitations}

This retrospective study combined two large prospectively collected cohorts from interventional trials conducted at two hospitals with inclusion of a representative and unselected cohort presenting around the clock, all days of the week, which is a major strength of the study. This study has several limitations. Patients with missing data were not included in the analyses, potentially causing a selection bias within the different risk assessment models investigated (i.e. patients with missing biomarkers (no blood analysis done) might be healthier than patients who had blood tests). Furthermore, although the missing data might not affect the comparison between age groups, the large difference in missing data (ADAPT missing in 50.3\%, CTA: 65.9\%, vital signs; $43.1 \%$, biomarkers: $38.7 \%$, and suPAR is missing in $85.0 \%$ of the total cohort) in the investigated models makes comparison of the risk assessment models difficult and should be interpreted with caution. Therefore, these results are only suitable for hypothesis generation. Measurement of vital signs are associated with some uncertainty, the temperature was measured using an ear thermometer, which might lead to underestimation of the actual temperature. All vital signs were recorded by hand and later entered manually in the database, which increases the risk of errors. The biomarker-based model in our study is based on the actual measurement value of the individual biomarkers, and this is not easy to implement in the clinical setting. Additionally, it is currently unknown whether they will have an impact when used as prognostic tools. This is also the case, regarding the experimental biomarker suPAR. This nonspecific marker of immune activation have previously demonstrated strong prognostic abilities, but only one trial has prospectively studied suPAR as a risk stratification tool and found no effect on mortality [26]. The suPAR level was only available in a small proportion of the total cohort and analysis of suPAR is not routinely performed in the ED, making it unavailable in most places or associated with increased costs. The investigated patients represent a subgroup of ED patients and the results might not be transferrable to different settings or in patients without need for blood sampling. Further research in the use this biomarker is required, before it can be considered for routine clinical use. The accuracy of the investigated triage algorithms might be artificially lower in the analyses, as a well-performing triage possibly translates to faster treatment of risk patients, hence lowering the risk of mortality. Furthermore, when the triage categories are used in the logistic models with only four levels, there is a limitation on the calculated AUC. Finally, we have not included comorbidity or diagnoses in our analyses, which would allow further stratification and precision in the models.

\section{Conclusion}

The predictive ability of mortality in acutely admitted patients was lower in older patients compared to middle-aged patients in all investigated models, therefore modifications for age should be considered when constructing risk assessment model for use in the emergency department. Models based on vital signs or routine biomarkers provided the best models for prediction of 7-day mortality.

\section{Additional file}

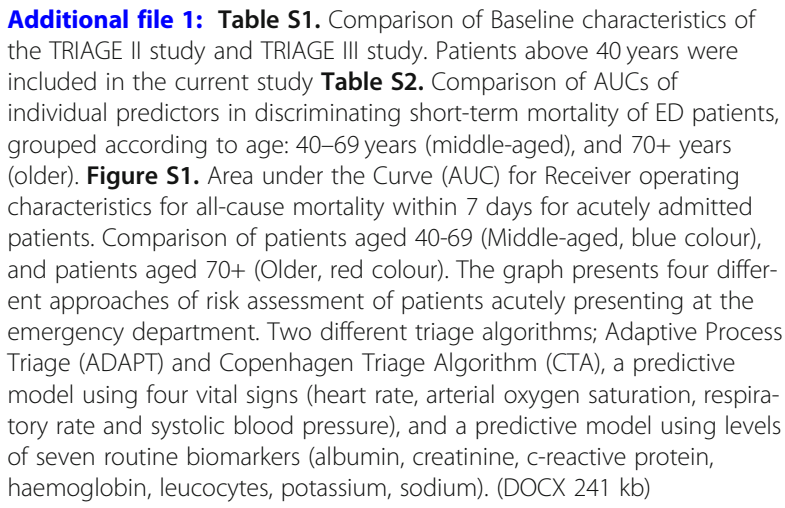

Additional file 1: Table S1. Comparison of Baseline characteristics of the TRIAGE II study and TRIAGE III study. Patients above 40 years were included in the current study Table S2. Comparison of AUCs of individual predictors in discriminating short-term mortality of ED patients, grouped according to age: 40-69 years (middle-aged), and 70+ years (older). Figure S1. Area under the Curve (AUC) for Receiver operating characteristics for all-cause mortality within 7 days for acutely admitted patients. Comparison of patients aged 40-69 (Middle-aged, blue colour) and patients aged $70+$ (Older, red colour). The graph presents four different approaches of risk assessment of patients acutely presenting at the emergency department. Two different triage algorithms; Adaptive Process Triage (ADAPT) and Copenhagen Triage Algorithm (CTA), a predictive model using four vital signs (heart rate, arterial oxygen saturation, respiratory rate and systolic blood pressure), and a predictive model using levels of seven routine biomarkers (albumin, creatinine, c-reactive protein, haemoglobin, leucocytes, potassium, sodium). (DOCX $241 \mathrm{~kb}$ )

\section{Abbreviations}

ADAPT: Adaptive Process Triage; AUC: Area under the curve; Cl: Confidence interval; CRP: C-reactive protein; CTA: Copenhagen Triage algorithm;

ED: Emergency department; suPAR: Soluble urokinase plasminogen activator receptor

\section{Acknowledgements}

The authors would like to extend our gratitude to the Departments of Clinical Biochemistry at Herlev Hospital and Bispebjerg Hospital for their professional assistance and support. Further, the corresponding author is grateful for valuable advice from Erik Kjøller and Theis Lange. 
Funding

None.

\section{Availability of data and materials}

The dataset supporting the conclusion of this article is available from the corresponding author upon reasonable request and conditioned by acceptance of a formal request by the Danish Data Protection Agency according to Danish law.

\section{Authors' contributions}

MS and KI designed the study. MS conducted the statistical analyses, created the figures, and wrote the first draft of the manuscript. All authors contributed substantially to the interpretation of the data, and results, revisions, and approved the final version of the manuscript.

\section{Ethics approval and consent to participate}

The TRIAGE studies were approved by Danish Patient Safety Authority instead of patient consent, in accordance with Danish legislation. All processing of personal data followed national guidelines and was approved by the Danish Data Protection Agency. Both TRIAGE studies were presented to the regional independent ethics committee, who decided that no consent were required in accordance with Danish law.

\section{Consent for publication}

Not applicable.

\section{Competing interests}

MS and LJHR have received funding for travel from ViroGates A/S. JE-O is named as an inventor in patents for the use of suPAR as a prognostic biomarker. The patents are owned by Copenhagen University Hospital, Amager and Hvidovre, Denmark, and are licensed to ViroGates A/S. JE-O is a cofounder, shareholder, and CSO of ViroGates A/S. The remaining authors have no conflicts of interest to declare.

\section{Publisher's Note}

Springer Nature remains neutral with regard to jurisdictional claims in published maps and institutional affiliations.

\section{Author details}

'Department of Cardiology, Herlev and Gentofte Hospital, University of Copenhagen, Herlev Ringvej 75, DK-2730 Herlev, Denmark. ${ }^{2}$ Department of Internal Medicine and Geriatrics, Herlev and Gentofte Hospital, University of Copenhagen, Herlev, Denmark. ${ }^{3}$ Clinical Research Centre, Amager and Hvidovre Hospital, University of Copenhagen, Hvidovre, Denmark. ${ }^{4}$ The Danish Heart Foundation, Copenhagen, Denmark. ${ }^{5}$ Department of Emergency Medicine, Bispebjerg Hospital, University of Copenhagen, Copenhagen, Denmark. ${ }^{6}$ Department of Health, Science and Technology, Aalborg University Hospital, Aalborg, Denmark. ${ }^{7}$ Department of Cardiology and Epidemiology/Biostatistics, Aalborg University Hospital, Aalborg, Denmark. ${ }^{8}$ Department of Anaesthesia, Centre of Head and Orthopaedics, Rigshospitalet, University of Copenhagen, Copenhagen, Denmark.

Received: 12 September 2018 Accepted: 9 May 2019

Published online: 16 May 2019

\section{References}

1. Guttmann A, Schull MJ, Vermeulen MJ, Stukel TA. Association between waiting times and short term mortality and hospital admission after departure from emergency department: population based cohort study from Ontario, Canada. BMJ. 2011;342(jun01 1):d2983.

2. Madsen F, Ladelund S, Linneberg A. High levels of bed occupancy associated with increased inpatient and thirty-day hospital mortality in Denmark. Health Aff. 2014;33:1236-44.

3. Farrohknia N, Castrén M, Ehrenberg A, Lind L, Oredsson S, Jonsson H, et al. Emergency department triage scales and their components: a systematic review of the scientific evidence. Scand J Trauma Resusc Emerg Med. 2011; 19:42.

4. Samaras N, Chevalley T, Samaras D, Gold G. Older patients in the emergency department: a review. Ann Emerg Med. 2010;56:261-9.
5. Pines JM, Mullins PM, Cooper JK, Feng LB, Roth KE. National trends in emergency department use, care patterns, and quality of care of older adults in the United States. J Am Geriatr Soc. 2013;61:12-7.

6. Carpenter CR, Shelton E, Fowler S, Suffoletto B, Platts-Mills TF, Rothman RE, et al. Risk factors and screening instruments to predict adverse outcomes for undifferentiated older emergency department patients: a systematic review and meta-analysis. Acad Emerg Med. 2015;22:1-21.

7. Aminzadeh F, Dalziel WB. Older adults in the emergency department: a systematic review of patterns of use, adverse outcomes, and effectiveness of interventions. Ann Emerg Med. 2002;39:238-47.

8. Djärv T, Castrén M, Martenson L, Kurland L. Decreased general condition in the emergency department: high in-hospital mortality and a broad range of discharge diagnoses. Eur J Emerg Med. 2015;22:241-6.

9. Gray LC, Peel NM, Costa AP, Burkett E, Dey AB, Jonsson PV, et al. Profiles of older patients in the emergency department: findings from the interrai multinational emergency department study. Ann Emerg Med. 2013;62:467-74.

10. Chester JG, Rudolph JL. Vital signs in older patients: age-related changes. J Am Med Dir Assoc. 2011;12:337-43.

11. Lamantia MA, Stewart PW, Platts-Mills TF, Biese KJ, Forbach C, Zamora E, et al. Predictive value of initial triage vital signs for critically ill older adults. West J Emerg Med. 2013;14:453.

12. Kuriyama A, Urushidani S, Nakayama T. Five-level emergency triage systems: variation in assessment of validity. Emerg Med J. 2017;34:703-10.

13. Beglinger B, Rohacek M, Ackermann S, Hertwig R, Karakoumis-Ilsemann J, Boutellier S, et al. Physician's first clinical impression of emergency department patients with nonspecific complaints is associated with morbidity and mortality. Medicine (United States). 2015;94:e374.

14. Brabrand M, Hallas J, Knudsen T. Nurses and physicians in a medical admission unit can accurately predict mortality of acutely admitted patients: a prospective cohort study. PLoS One. 2014;9(7):e101739.

15. Barfod C, Lauritzen MMP, Danker JK, Sölétormos G, Forberg JL, Berlac PA, et al. Abnormal vital signs are strong predictors for intensive care unit admission and in-hospital mortality in adults triaged in the emergency department - a prospective cohort study. Scand J Trauma Resusc Emerg Med. 2012;20:28.

16. Olsson T, Terent A, Lind L. Rapid emergency medicine score can predict long-term mortality in nonsurgical emergency department patients. Acad Emerg Med. 2004;11:1008-13.

17. Oh J, Kim SH, Park KN, Oh SH, Kim YM, Kim HJ, et al. High-sensitivity Creactive protein / albumin ratio as a predictor of in- hospital mortality in older adults admitted to the emergency department. Clin Exp Emerg Med. 2017:4:19-24.

18. Socorro García A, De La Fuente HI, Baztán JJ. Serum albumin and total cholesterol as prognostic factors of mortality in very old patients hospitalized by acute illness. Eur Geriatr Med. 2015;6:442-6.

19. Kristensen M, Iversen AKS, Gerds TA, Østervig R, Linnet JD, Barfod C, et al. Routine blood tests are associated with short term mortality and can improve emergency department triage: a cohort study of $>12,000$ patients. Scand J Trauma Resusc Emerg Med. 2017;25:115.

20. Kutz A, Hausfater P, Amin D, Amin A, Canavaggio P, Sauvin G, et al. The TRIAGE-ProADM score for an early risk stratification of medical patients in the emergency department - development based on a multi-national, prospective, observational study. PLoS One. 2016;11:1-17.

21. Nickel $\mathrm{CH}$, Messmer AS, Geigy N, et al. Stress markers predict mortality in patients with nonspecific complaints presenting to the emergency department and may be a useful risk stratification tool to support disposition planning. Acad Emerg Med. 2013;20(7):670-9

22. Iversen K, Gotze JP, Dalsgaard M, Nielsen H, Boesgaard SSS, Bay M, et al. Risk stratification in emergency patients by copeptin. BMC Med. 2014;12:80.

23. Rasmussen LJH, Ladelund S, Haupt TH, Ellekilde G, Poulsen JH, Iversen K, et al. Soluble urokinase plasminogen activator receptor (suPAR) in acute care: a strong marker of disease presence and severity, readmission and mortality. A retrospective cohort study. Emerg Med J. 2016;33:769-75.

24. Nayak RK, Allingstrup M, Phanareth K, Kofoed-Enevoldsen A. suPAR as a biomarker for risk of readmission and mortality in the acute medical setting. Dan Med J. 2015;62:1-4.

25. Haupt TH, Petersen J, Ellekilde G, Klausen HH, Thorball CW, Eugen-Olsen J, et al. Plasma suPAR levels are associated with mortality, admission time, and Charlson comorbidity index in the acutely admitted medical patient: a prospective observational study. Crit Care. 2012;16:R130. 
26. Schultz M, Rasmussen LJH, Andersen MH, Stefansson JS, Falkentoft A, Alstrup $\mathrm{M}$, et al. Use of the prognostic biomarker suPAR in the emergency department improves risk stratification but has no effect on mortality: a cluster-randomized clinical trial (TRIAGE III). Scand J Trauma Resusc Emerg Med. 2018;26:69

27. Hasselbalch RB, Plesner LL, Pries-Heje M, Ravn L, Lind M, Greibe R, et al. The Copenhagen triage algorithm: a randomized controlled trial. Scand J Trauma Resusc Emerg Med. 2016;24:123.

28. Sandø A, Schultz M, Eugen-Olsen J, Rasmussen LS, Køber L, Kjøller E, et al. Introduction of a prognostic biomarker to strengthen risk stratification of acutely admitted patients: rationale and design of the TRIAGE III cluster randomized interventional trial. Scand J Trauma Resusc Emerg Med. 2016;24:100.

29. Conroy SP, Stevens T, Parker SG, Gladman JRF. A systematic review of comprehensive geriatric assessment to improve outcomes for frail older people being rapidly discharged from acute hospital: "interface geriatrics". Age Ageing. 2011;40:436-43. https://doi.org/10.1093/ageing/afr060.

30. de Groot B, Stolwijk F, Warmerdam M, Lucke JA, Singh GK, Abbas M, et al. The most commonly used disease severity scores are inappropriate for risk stratification of older emergency department sepsis patients: an observational multi-centre study. Scand J Trauma Resusc Emerg Med. 2017; 25:91. https://doi.org/10.1186/s13049-017-0436-3.

31. Hubbard RE, Peel NM, Scott IA, Martin JH, Smith A, et al. Polypharmacy among inpatients aged 70 years or older in Australia. MJA. 2015;202. https:// doi.org/10.5694/mja13.00172

32. Lynge E, Sandegaard JL, Rebolj M. The Danish national patient register. Scand J Public Health. 2011;39(7_suppl):30-3.

33. Schmidt M, Schmidt SAJ, Sandegaard JL, Ehrenstein V, Pedersen L, Sørensen $\mathrm{HT}$. The Danish national patient registry: a review of content, data quality, and research potential. Clin Epidemiol. 2015;7:449-90.

34. Iversen AKS, Kristensen M, Østervig R, Forberg JL, Schou M, Iversen K. No evidence that formalized triage is superior to informally structured triage. Ugeskr Laeger. 2015;20:177.

35. Nordberg M, Lethvall $S$, Castrén $M$. The validity of the triage system ADAPT. Scand J Trauma Resusc Emerg Med. 2010;18(Suppl 1):P36.

36. Göransson KE, Von Rosen A. Interrater agreement: a comparison between two emergency department triage scales. Eur J Emerg Med. 2011;18:68-72.

37. Schuetz P, Hausfater P, Amin D, Amin A, Haubitz S, Faessler L, et al. Biomarkers from distinct biological pathways improve early risk stratification in medical emergency patients: the multinational, prospective, observational TRIAGE study. Crit Care. 2015;19:377.

38. DeLong ER, DeLong DM, Clarke-Pearson DL. Comparing the areas under two or more correlated receiver operating characteristic curves: a nonparametric approach. Biometrics. 1988:44:837-45.

39. R Core Team. R: a language and environment for statistical computing. Vienna: R Foundation for Statistical Computing; 2017. https://www.r-project. org/. Accessed 9 Apr 2018

40. Robin X, Turck N, Hainard A, Tiberti N, Lisacek F, Sanchez, Jean-Charles Müller M. pROC: an open-source package for R and S+ to analyze and compare ROC curves. BMC Bioinformatics. 2011:12:77.

41. Ljunggren $M$, Castrén $M$, Nordberg $M$, Kurland $L$. The association between vital signs and mortality in a retrospective cohort study of an unselected emergency department population. Scand J Trauma Resusc Emerg Med. 2016;24:21.

42. Churpek MM, Yuen TC, Winslow C, Hall J, Edelson DP. Differences in vital signs between elderly and non-elderly patients prior to ward cardiac arrest Matthew. Crit Care Med. 2015;344:1173-8.

43. Brabrand M, Folkestad L, Clausen NG, Knudsen T, Hallas J. Risk scoring systems for adults admitted to the emergency department: a systematic review. Scand J Trauma Resusc Emerg Med. 2010;18:8.

44. Baumann MR, Strout TD. Triage of geriatric patients in the emergency department: validity and survival with the emergency severity index. Ann Emerg Med. 2007;49:234-40.

45. Platts-Mills TF, Travers D, Biese K, McCall B, Kizer S, LaMantia M, et al. Accuracy of the emergency severity index triage instrument for identifying elder emergency department patients receiving an immediate life-saving intervention. Acad Emerg Med. 2010;17:238-43.

46. Grossmann FF, Zumbrunn T, Frauchiger A, Delport K, Bingisser R, Nickel $\mathrm{CH}$. At risk of undertriage? Testing the performance and accuracy of the emergency severity index in older emergency department patients. Ann Emerg Med. 2012;60:317-25. https://doi.org/10.1016/j.annemergmed.2011. 12.013 .
47. Smith GB, Prytherch DR, Schmidt PE, Featherstone PI, Kellett J, Deane B, et al. Should age be included as a component of track and trigger systems used to identify sick adult patients? Resuscitation. 2008;78:109-15.

48. Subbe CP, Kruger M, Rutherford P, Gemmel L. Validation of a modified early warning score in medical admissions. QJM. 2001;94:521-6.

49. Brabrand M, Lassen AT, Knudsen T, Hallas J. Seven-day mortality can be predicted in medical patients by blood pressure, age, respiratory rate, loss of independence, and peripheral oxygen saturation (the PARIS score): a prospective cohort study with external validation. PLoS One. 2015;10:1-12

50. Coslovsky M, Takala J, Exadaktylos AK, Martinolli L, Merz TM. A clinical prediction model to identify patients at high risk of death in the emergency department. Intensive Care Med. 2015:1029-36. https://doi.org/10.1007/ s00134-015-3737-x.

51. Goodacre S, Turner J, Nicholl J. Prediction of mortality among emergency medical admissions. Emerg Med J. 2006:23:372-5.

52. Le Gall J, Lemeshow S, Saulnier F. A new simplified acute physiology score (SAPS II) based on a European/north American multicenter study. JAMA. 1993;270:2957-63.

53. Campbell SE, Seymour DG, Primrose WR. A systematic literature review of factors affecting outcome in older medical patients admitted to hospital. Age Ageing. 2004;33:110-5.

\section{Ready to submit your research? Choose BMC and benefit from:}

- fast, convenient online submission

- thorough peer review by experienced researchers in your field

- rapid publication on acceptance

- support for research data, including large and complex data types

- gold Open Access which fosters wider collaboration and increased citations

- maximum visibility for your research: over $100 \mathrm{M}$ website views per year

At $\mathrm{BMC}$, research is always in progress.

Learn more biomedcentral.com/submissions 